\title{
Agent-Based Services for Building Markets in Distributed Energy Environments
}

\author{
I. Lopez-Rodriguez ${ }^{1}$ and M. Hernandez-Tejera ${ }^{1}$ \\ ${ }^{1}$ SIANI Universitary Institute \\ University of Las Palmas de Gran Canaria
}

Scientific and Technological Park - Campus Universitario de Tafira, 3017 Las Palmas de Gran Canaria (Spain)

Phone number: +(34)928727614,+(34)928458744,/Fax: +(34)928458711 e-mail: ijlopez@ siani.es, fmhernandez@ siani.es

\begin{abstract}
The safe integration of renewable energy sources requires distributed energy environments provided with reactive and dynamic management systems. To this end, recent works propose creating energy micromarkets in which producers and consumers are capable of negotiating their interests by using autonomous software entities. Despite intelligent agents are widely accepted as a suitable technology for undertaking this task, we point out that their adoption entails important risks. Specifically, this paper highlight a set of technological and structural challenges that implies the integration of agents with distributed energy networks and, as a result, it concludes that new contributions are necessary in the modelling field for building more flexible, reactive and scalable networks. With this aim, we put forward a new agent-based and servicesoriented model called Agency Services that, apart from overcoming most of the challenges faced by the current proposals, gives new capabilities.
\end{abstract}

\section{Keywords}

Energy Market, Intelligent Agent, Distributed Energy, Renewable Energy Sources, Microgrid.

\section{Introduction}

The reactive, intermittent and distributed nature of Renewable Energy Sources (RES) makes it difficult to integrate them into the electrical grid, which is based on vertical and poorly reactive control systems. In this context, RES not only lose much of their potential, but they also become a dangerous technology for grid stability.

In order to integrate the new energy sources in a safe way, as well as to exploit their full potential, it is required the creation of distributed energy environments capable of reacting on real-time to both availability of sources and demand. In this sense, the community devoted to the energy systems is currently working on the design of smart management systems based on market principles. Specifically, they work on the idea of creating energy micromarkets in which producers and consumers are able to insure energy balance by negotiating and coordinating their actions. This model, besides providing the reactivity required, would ensure more competitive energy prices.
Many studies have identified Intelligent Agents as a suitable technology for meeting the needs of these energy micromarkets. However, despite their well-known ability for the management of distributed environments, we believe that it is still a difficult task to build reliable grids based on autonomous agents. We have identified that adopting this technology actually entails many structural and technological challenges that, in spite of the importance of the context, have not yet been studied in depth.

Therefore, in order to get distributed electrical grids that are flexible and efficient, new contributions are still necessary on the modelling field. In response to these requirements, we have designed a services-oriented model that facilitates the creation of energy micromarkets based on autonomous intelligent agents, which we term Agency Services for Energy Management.

The remainder of this article is structured as follows. Section 2 describes recent work in the field of distributed energy management and the concept of Energy Micromarket. Section 3 gives an overview of intelligent agents. Section 4 comments the technological and structural challenges that imply adding intelligent agents to the distributed energy management. Section 5 describes the application of the Agency Services model for building energy micromarkets, and Section 6 concludes.

\section{Energy Micromarkets}

With the aim of facilitating the RES integration and initiating a gradual transition to a distributed network, recent works propose the creation of autonomous local areas in the electrical grid ([1]). They are based on the concept of Virtual Utility ([2]) and are conceived as a self-controlled enclosed cell of the distribution grid that is composed of distributed sources, dispatchable loads and storage systems. These cells are characterized by smart control systems that are able to provide energy balance in a dynamic way, thus shaping an optimum environment for RES integration. The main aim of this 
model is to build a grid composed of many linked selfsupplied cells capable of exchanging energy between them. In this way, it is expected to satisfy most of the energy demand in future.

In this setting, the Microgrid concept represents one of the most representative lines of work ([3]). Microgrids operate mostly connected to the distribution grid, and among their principal features, it stands out their ability to be automatically transferred to islanded mode in case of fault in the main grid. From the point of view of the System Operator, a microgrid is another point of the network whose production and demand can be controlled. In the future, it is expected that microgrids can also provide ancillary services to the main grid by supplying energy and by modulating the demand of their loads when necessary.

Microgrids, and distributed energy environments in general, have to meet economic, heat load, environmental and legislative constraints. Therefore, apart from the fast electrical control systems, they require building an intelligent global control system. This is called Energy Manager (EM) and aims to optimize cell's energy cost through planning, coordinating and supervising the activity of all resources ([4]). The EM is a secondary regulation control system that makes short-term plans based on factors such as: (1) specific features of the supply devices; (2) amount of load that can be modulated and shed; (3) amount of energy that can be stored; (4) demand and weather forecasts; (5) energy prices; (6) or current legislation.

Note that the EM does not necessarily imply the presence of a physical device. The EM is primarily a concept, so that it can be implemented using the most simple and archaic methods, like the control done by hand, or the most modern and sophisticated ones, like distributed systems based on concepts and techniques belonging to the artificial intelligence field.

Building an EM is a difficult task. In practice, with the aim of testing the feasibility of the microgrid concept, the EM has been traditionally implemented as a centralized module. In this line, the community usually proposes the use of optimization algorithms ([5]). However, this technique can be considered neither efficient nor scalable because: (1) it entails a heavy computational load that forces to simplify the model; (2) its cost increases exponentially with the size of the model; (3) it is not able to solve problems involving stochastic and nonlinear variables in an effective way. Even though there are also proposals based on neural networks and fuzzy logic ([6]), the adoption of centralized solutions is not recommended in general, as they grant neither autonomy of the users nor flexibility required by distributed grids.

To overcome all the above challenges, it has been recently proposed to adopt hierarchical and decentralized control systems. In this line of work, the European research project CRISP ([7]) describes a model where autonomous software agents bargain and make plans on behalf of the supply and demand devices. The model is called SDM [Supply and Demand Matching] ([8]) and, unlike techniques such as DSM (Demand Side Management) or DRR (Demand Response Resources), it guarantees autonomy of producers.

On a smaller scale, the SDM model emulates the mechanics of traditional energy markets. Through negotiations, it is decided: the amount of energy that each unit must produce and consume, and the period during which they can do that. The SDM model proposes to create energy micromarkets that would be highly reactive, instantiated on demand, and that would span a short time horizon (commonly shorter than 15 minutes).

The idea of managing distributed grids by using autonomous entities capable of dynamically bargaining the amount of energy they can produce and consume is considered one of the most promising options for RES integration. Essentially, its advantages include:

- It guarantees producers and consumers' autonomy, so that they can participate according to their aims.

- It helps to interiorize energy prices, thus giving competitive advantage to the RES.

- It attracts new investments for the RES field due to both the users' ability to negotiate, and the presence of competitive prices.

- It helps to exploit RES capacity due to the reactive and flexible nature of the context.

- It improves the stability of the grid due to the better integration of the RES.

\section{Intelligent Agents}

The complexity of managing distributed energy environments and the nature of future requirements over electrical grid, force the adoption and integration of new Information and Communications Technologies (ICT). Specially, they are necessary technological solutions in accordance with the distributed nature of the new energy sources and able to increase the autonomy of the users.

The SDM model demands placing autonomous software programs at each supply and consumption point in the grid. The goal of these programs is to represent the interests of the user in energy micromarkets, and coordinate devices' actions in order to guarantee global goals achievement such as grid stability, power quality or supply security. Intelligent Agents (IA) have all of these skills and therefore they are considered a suitable technology to develop the future electrical grids ([9] [14]).

In particular, IAs are autonomous entities capable to develop flexible action planning in a certain environment to achieve well defined goals ([15]). By extension, Multiagent Systems are configurations and environments where multiple agents interact. Main features of IAs are:

- Goal orientation: Agents are designed to achieve specific and well-defined goals that include: global goals related to the environment where they are 
embedded, and local goals related to the interests they represent.

- Autonomy: Agents are capable to decide which actions must be applied to achieve defined goals.

- Social ability: Agents communicate with each other and with people through interfaces and standard communication languages.

- Reactivity: Agents adapt their response to changes in the environment they inhabit.

- Proactivity: Agents develop action plans and take initiatives related to the global and local goals to be satisfied.

In the last years, the distributed artificial intelligence and multiagent systems communities have developed algorithms, architectures and methodologies to solve problems in distributed environments by means of societies of autonomous agents. In this sense, the community devoted to the development of energy systems can make use of the experience of agency theory in topics such as setting up coalitions, plan elaboration, task coordination or negotiations ([16]). To this end, some conceptually similar areas, like the one devoted to the computational grids, have already taken advantage of this experience ([17]).

Thanks to their skills, agents placed at the supply and consumption points make the micromarkets more autonomous and dynamic. Moreover, agents facilitate the creation of an ICT infrastructure more open, flexible, scalable, and fault tolerant.

\section{Challenges of adding Intelligent Agents}

Although IAs are considered a suitable technology for future electrical grids, its inclusion faces important challenges that have not been properly considered. These can be classified as structural or technological challenges.

\subsection{Structural Challenges}

It is assumed that in the SDM model each device has associated an agent. In a real setting, this agent runs in a local device similar to Advanced Metering Infrastructure (AMI). For example, in the CRISP project, the E-box device was developed to carry out tests.

From a structural point of view, the idea of running the agent that represents the user in a local device is far from being a flexible solution. The reason is that, if the agent reconfiguration or replacement is required, these tasks must be done by hand. Furthermore, they are tasks that will be probably required, since it is not realistic to consider software agents, or whatever software, to be error free or be ready to react to all possible events in an electrical grid setting. It may also be necessary to configure the agent because of other reasons like: the development of more effective negotiation algorithms, error debugging in agent behaviours, the need to adapt agents to specific and new circumstances, or the replacement of hardware by other device with better performances.
In this situation, maintenance tasks of medium and big size networks can be slow and expensive. Therefore, it is essential to develop models where these tasks can be done in a suitable way in order to achieve flexible, dynamic and effective electrical grids.

\subsection{Technological challenges}

Concerning to the management, distributed electrical grids can be considered as Virtual Organizations (VO). Specifically, VO is a name used for situations where distributed entities collaborate and share resources to meet their own goals ([18]). Although VOs are widely used to model and solve different kinds of problems, there are no references related to their use in electrical grids. However, the analysis of electrical grids as VOs allows taking advantage of all the experience accumulated in similar areas like computational grids, and also very important, to easily identify the challenges that will be faced in a near future.

One of the main challenges faced by VOs is related to the creation stage ([19]), in which the following tasks must be accomplished: business opportunity identification, action plan development and suitable partner selection. In the traditional model (Fig. 1), all these tasks are carried out by the local agents, which must be properly qualified in order for the VO to become successful. In general, models in which the partners are well known, and in which it is possible to previously certify their efficiency and behaviour, are accepted as suitable models to cancel the risks of the creation stage.

In the future, users will need to access weather forecast services in order to estimate with precision the energy their devices will produce and consume. Having this information will be essential for participation in energy markets. In the traditional model, the local agent is in charge of accessing the external forecast services, as well as of processing the information according to the features of the local resources it represents. However, this task turns out complex and heavy for a local control device that is supposed to be simple and installed on a large scale.

In distributed energy environments, it is also considered the option that several supply units can make coalitions and participate in the market as a single unit, which is commonly known as a Virtual Power Plant. In the models proposed up to now, this responsibility would fall on the local agents, which should dynamically coordinate all their actions. Again, this could turn out rather complex for a local control device.

In the case of electrical grids, it is essential to cancel the challenges pointed out previously. Otherwise, grid security and efficient energy exploitation of RES will be affected. 


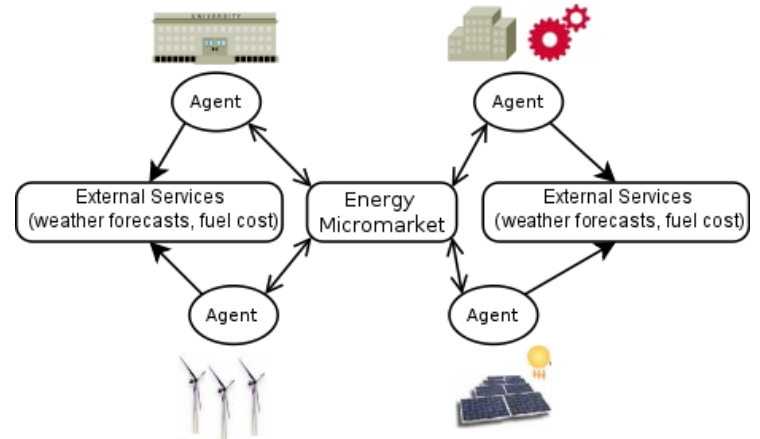

Fig. 1. Energy micromarket based on agents.

\section{Energy markets based on Agency Services}

In order to provide solutions to all the previous challenges, then we put forward an agent-based architectonic model specifically designed for building large virtual organizations. The model is called Agency Services ([20]) and its adoption would facilitate the creation of energy micromarkets in a realistic way.

\subsection{Description}

The main aim of the Agency Services model is to free local control devices (and their corresponding agents) from the complexity that would involve their direct participation in next energy micromarkets.

To achieve this, the new model proposes the instantiation of additional intermediary agents in the ICT infrastructure, which after being contracted as services will participate in the energy micromarkets on behalf of the users. These agents would work as advanced external brokers carrying out tasks related to planning, bargaining and cooperation.

In spite of the addition of an external broker agent, the presence of a local agent is still necessary. Specifically, for the client side the Agency Services model proposes the instantiation of a very light agent capable of monitoring the activity of the local devices. In this regard, each local agent is related to a broker agent of the ICT infrastructure, and a broker agent is in charge of representing one or more local agents in the micromarket.

Interactions between the local agent and the broker agent can be summarized in the following lines:

- The local agent monitors local devices activity and transmits this information to the broker agent.

- In accordance with the information sent by the local agent, the broker agent negotiates on behalf of the user in the energy market. When the negotiation is over, the broker agent informs the local agent about the dispatch directives to be applied to the local devices.

- The local agent supervises the scheduled plan and informs the broker agent of any unexpected event.
Likewise, the broker agent lets the local agent know whether the initial plan has to be changed.

One of the most remarkable features of the Agency Services model is that it uses the latest information technologies for remotely transmitting and remotely instantiating the local agent on the client. That is, the local agent can be dynamically reloaded and configured when necessary, thereby getting a highly reactive context. These processes are facilitated by the small size of the local agent.

The Agency Services can be provided by firms specialized in the energy sector, which we term Agency Services Provider for Energy Management (thereafter called ASPEM). When a user contracts its services, the ASPEM is responsible for both providing the broker agent in the ICT infrastructure and remotely loading the local agent in the client. Both agents are aware of each other's existence and are capable of communicating with each other.

According to the Agency Services model, the energy micromarket would be instantiated in a ICT infrastructure, in which ASPEMs deploy the broker agents that negotiate between them the energy that will be produced, consumed and stored (in accordance with the information transmitted by the local agents about their local devices). The System Operator would be responsible for providing that ICT infrastructure, as well as for approving the ASPEMs that can deploy their broker agents. Moreover, nothing prevents the System Operator from providing its own agency services and work as an ASPEM.

Taking advantage of their technological capabilities, ASPEMs are able to gain access to weather forecast services of third parties, or even provide their own ones. In general, ASPEMs can process weather information more easily than local agents can, as well as provide this information directly to the broker agents for their participation in the markets. Likewise, ASPEMs can facilitate the access to other useful services, such as those related to the fuel costs and the demand history. In general, the Agency Services model helps to build services-oriented grids.

In the new model, users will only need a local device able to run the local agent transmitted by the ASPEM. We term this device EAS-Box and it performs the following duties: monitors the activity of the local resources, transmits the needed information to the broker agent, and runs the directives received from it. In this regard, the Java technology has all the necessary means for remotely transmitting software and for remotely running it ([21]). Therefore, EAS-Box might be based on any existent device capable of launching Java programs and that is provided with connection interfaces.

In order to guarantee the autonomy of the users, ASPEMs can provide powerful web-based user interfaces that clients can use to send their directives to the broker agents. Thus, in the Agency Services model it is not 
necessary for the user to interact with the device where the local agent runs, which commonly provides a limited interface.

Following the ideas expressed above, Figure 2 shows a sketch of the new proposed model for the creation of energy markets in distributed energy environments.

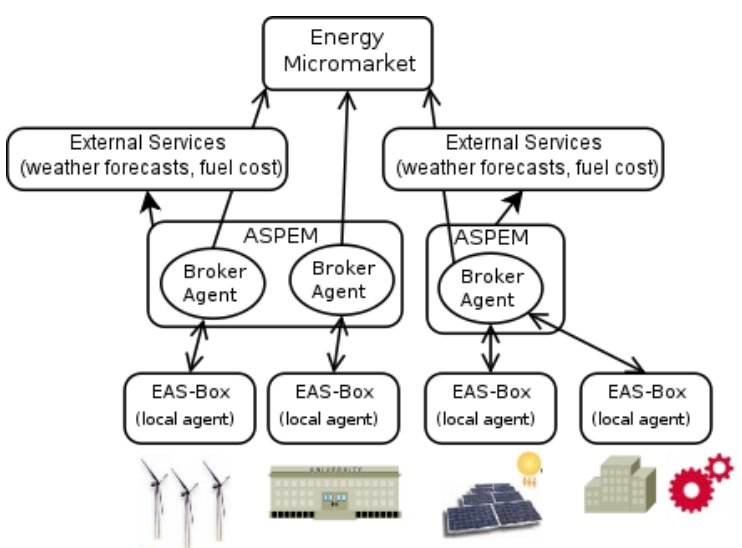

Fig. 2. Energy micromarket based on the Agency Services model.

\subsection{Advantages}

Agency Services for Energy Management finds solutions to the structural and technological challenges pointed out in the section 4 of this document.

Regarding the structural aspect, the advantages of adopting the new model are:

- Flexibility: ASPEMs can automatically update the software of both local and broker agents without requiring interacting with physical devices.

- Maintenance: Duties delegated to the local agent are limited. This makes possible to use physical devices in the client side that are cheaper and easier to install and maintain.

- Scalability: The responsibility for building larger and more sophisticated micromarkets falls on both the System Operator and ASPEMs, which are technological entities with the required means.

- Services Orientation: Apart from working as services providers, ASPEMs facilitate the interaction with other useful services for energy management that might be provided by third parties.

On the technological side, the advantages of the new model are:

- Reliability: All agents participating in the micromarkets are provided by ASPEMs, which are firms previously approved by the System Operator. This guarantees that the activity of the broker agents does not endanger the stability of the system with selfish or anti-social behaviors, thereby avoiding the typical risks of the creation stage of VOs.

- Virtual Production: A broker agent can act on representation of many physically distributed supply units (many local agents), thereby shaping Virtual Power Plants.

- Abstraction: The transfer of the most complex tasks to the ASPEMs, together with the simplicity of the technologies used on the client, facilitates users to automate their participation in energy micromarkets.

- Autonomy: The user keeps the option of sending directives to the broker agent by using the interfaces provided by the ASPEM. These interfaces are powerful and can be accessible from heterogeneous sources such as computers, cell phones or PDAs.

\section{Conclusions}

The creation of energy micromarkets in which producers and consumers are represented by software agents constitute a promising option for the gradual integration of the renewable energy sources. However, as has been shown, the formation of agent-based distributed energy environments faces structural and technological challenges that must be overcome in order to achieve a flexible and dynamic context. Aware of this need, this paper put forward an architectonic model called Agency Services for Energy Management. The model frees the local devices from the complexity that entails the direct participation of autonomous entities in markets, provides solutions to the challenges that imply the presence of software agents at the production and consumption points, and build a context able to interact with other useful services for energy management in distributed environments. Agency Services for Energy Management are presented as a suitable model for building a grid composed of linked self-supplied cells, which insure energy balance by using mechanisms based on market principles.

\section{References}

[1] I.G. Kamphuis, P. Carlsson, C.J. Warmer, J.C.P. Kester and J.K. Kok, "Distributed Intelligence for Supply/Demand Matching to Improve Embedding of Distributed Renewable Energy Sources", in Proc. $2^{\text {nd }}$ International Conference on Critical Infrastructures (CRIS), 2004.

[2] D. Coll-Mayor, R. Picos and E. Garcia-Moreno. "State of the art of the virtual utility: the smart distributed generation network", International Journal of Energy Research, vol. 28, pp. 65-80, 2004.

[3] R. Lasseter, "Integration of Distributed Energy Resources. The CERTS Microgrid Concept", Technical Report, Consortium for Electric Reliability Technology Solutions, 2002.

[4] J. D. Kueck, R.H. Staunton, S.D. Labinov, B.J. Kirby, "Microgrid Energy Management System", Technical Report, Consortium for Electric Reliability Technology Solutions, 2003. 
[5] N. Hatziargyriou, A. Tsikalakis, J. Vlachogiannis, K. Papadogiannis, G. Kariniotakis, J.P. Lopes, J. Oyarzabal, C. Moreira, A. Madureira and I. Cobelo, "Microgrid central controller strategies and algorithms". Technical Report, Microgrids EU Research Project, 2004.

[6] G. Celli, F. Pilo, G. Pisano and G. Soma, "Optimal Participation of a Microgrid to the Energy Market with an Intelligent EMS", International Power Engineering Conference, pp. 663-668, 2006.

[7] CRISP EU Research Project. http://crisp.ecn.nl.

[8] R. Kamphuis, P. Carlsson, J. Kester and H. Akkermans, "Market-oriented online supply-demand matching", Technical Report, CRISP EU Research Project, 2004.

[9] S. Rahman, M. Pipattanasomporn and Y. Teklu, "Intelligent Distributed Autonomous Power Systems (IDAPS)", In Proc. IEEE PES Annual General Meeting, pp. 1-8, 2007.

[10] M. Pipattanasomporn, H. Feroze and S. Rahman, "MultiAgent Systems in a Distributed Smart Grid: Design and Implementation", In Proc. Power Systems Conference \& Exposition (PSCE), 2009

[11] L. Phillips, M. Link, R. Smith and L. Weiland, "Agentbased control of distributed infrastructure resources", Technical Report, Sandia National Laboratories, 2006.

[12] K. Kok, C. Warmer and R. Kamphuis, "The PowerMatcher: Multiagent control of electricity demand and supply", in Proc. AAMAS, pp. 75-82, 2005.

[13] A. Dimeas and N. Hatziargyriou, "Operation of a Multiagent System for Microgrid Control”, IEEE Transactions on Power Systems, vol. 20, pp. 1447-1455, 2005.

[14] J. Oyarzabal and J. Jimeno, "Agent based Micro Grid Management System", in Proc. Future Power Systems (FPS), pp. 16-18, 2005.

[15] M. Wooldridge, “Agent-Based Software Engineering”, IEE Proc. Software Engineering, vol. 144, pp. 26-37, 1997.

[16] T.W. Sandholm, "Multiagent Sytems: A modern approach to Distributed Artificial Intelligence", chapter Distributed Rational Decision Making, pp. 201-258, 1999.

[17] C. Kesselman, “Applications of Intelligent Agent Technology to The Grid", in Proc. Intelligent Agent Technology (IAT), pp. 1-4, 2004.

[18] M. R. Nami and D. Tavangarian, "Virtual Organization: A New Approach in IT", in Proc. 7th IEEE International Conference on Computer and Information Technology (ICCIT), pp. 93-98, 2007.

[19] Camarinha-Matos, "Virtual Organizations", chapter ICT Infrastructures for VO, pp. 83-103, 2006.

[20] I. Lopez-Rodriguez, M. Hernandez-Tejera, "Agency Services: An Agent-Based and Services-Oriented Model for Building Large Virtual Communities", in Proc. International Conference on Agents and Artificial Intelligence (ICAART), 2010.

[21] JXTA, JXTA Community, https://jxta.dev.java.net. 